\title{
Increasing access to reproductive health services through maternity waiting homes for women living farthest from a health facility in rural Zambia: a quasi-experimental study
}

Jody Lori ${ }^{1}$, Michelle Munro-Kramer ${ }^{1}$, Haiyin Liu ${ }^{1}$, Kathleen McGlasson ${ }^{2}$, Xingyu Zhang ${ }^{1}$, HaEun Lee ${ }^{1}$, Thandiwe Ngoma ${ }^{3}$, Jeanette Kaiser ${ }^{2}$, Misheck Bwalya ${ }^{3}$, Gertrude Musonda ${ }^{4}$, Isaac Sakala ${ }^{4}$, Joseph Perosky ${ }^{1}$, Rachel Fong ${ }^{2}$, Carol Boyd ${ }^{1}$, Parker Chastain ${ }^{2}$, Peter Rockers ${ }^{2}$, Davidson Hamer, $\mathrm{MD}^{5}$, Godfrey Biemba ${ }^{6}$, Taryn Vian² ${ }^{2}$, Rachael Bonawitz ${ }^{2}$, Nancy Lockhart ${ }^{1}$, and Nancy Scott ${ }^{2}$

${ }^{1}$ University of Michigan

${ }^{2}$ Boston University

${ }^{3}$ Right to Care

${ }^{4}$ Africare

${ }^{5}$ Boston University School of Public Health

${ }^{6}$ National Health Research Authority

October 12, 2020

\begin{abstract}
Objective: To report on the effectiveness of a standardized core Maternity Waiting Home (MWH) model to increase facility deliveries and access to reproductive health services among women living farthest from a health facility $(>10 \mathrm{~km})$ using facilitybased data. Design: Quasi-experimental design. Setting: Seven rural districts in Zambia. Population: Women delivering at 40 health facilities between June 2016 to August 2018. Methods: 20 intervention sites and 20 comparison sites were used to test if MWHs increased access to reproductive health services for women living in rural Zambia. The difference-in-differences (DID) methodology was used to examine the effectiveness of the core MWH model on our primary outcomes. Main Outcome Measures: Differences in the change from baseline to endline in the percentage of women who: 1) traveled greater than 10 $\mathrm{km}$ for delivery, (2) attended a postnatal visit at 6 days postpartum, and (3) were referred to a higher-level health facility between intervention and comparison group. Results: We detected a significant difference for the percentage of deliveries at intervention facilities with the core MWH model for all women living $>10 \mathrm{~km}$ away $(\mathrm{p}=0.03$ ), adolescent women ( $<18$ years) living $>10 \mathrm{~km}$ away $(\mathrm{p}=0.002)$, and primigravida women living $>10 \mathrm{~km}$ away $(\mathrm{p}=0.01)$. There were no significant differences for women attending a postnatal care visit at 6 days postpartum $(\mathrm{p}=0.07)$ or for women referred to the next level of care $(\mathrm{p}=0.29)$. Conclusion: The core MWH model was successful in reaching women with historically low rates of facility delivery, those living $>10 \mathrm{~km}$ from a healthcare facility, including adolescent women and primigravidas.
\end{abstract}

\section{Hosted file}

Main manuscript_BJOG.pdf available at https://authorea.com/users/366460/articles/486217increasing-access-to-reproductive-health-services-through-maternity-waiting-homes-forwomen-living-farthest-from-a-health-facility-in-rural-zambia-a-quasi-experimental-study 